\title{
Article
}

\section{Bell-Based Bernoulli Polynomials with Applications}

\author{
Ugur Duran $^{1} \oplus$, Serkan Araci ${ }^{2, *} \mathbb{1}$ and Mehmet Acikgoz ${ }^{3}$ \\ 1 Department of Basic Sciences of Engineering, Faculty of Engineering and Natural Sciences, Iskenderun \\ Technical University, TR-31200 Hatay, Turkey; mtdrnugur@gmail.com \\ 2 Department of Economics, Faculty of Economics, Administrative and Social Sciences, Hasan Kalyoncu \\ University, TR-27410 Gaziantep, Turkey \\ 3 Department of Mathematics, Faculty of Arts and Science, University of Gaziantep, \\ TR-27310 Gaziantep, Turkey; acikgoz@gantep.edu.tr \\ * Correspondence: serkan.araci@hku.edu.tr
}

check for updates

Citation: Duran, U.; Araci, S.; Acikgoz, M. Bell-Based Bernoulli Polynomials with Applications. Axioms 2021, 10, 29. https://doi.org/ $10.3390 /$ axioms 10010029

Academic Editor: Clemente Cesarano Received: 5 February 2021 Accepted: 24 February 2021 Published: 2 March 2021

Publisher's Note: MDPI stays neutral with regard to jurisdictional clai$\mathrm{ms}$ in published maps and institutional affiliations.

Copyright: $\odot 2021$ by the authors. Licensee MDPI, Basel, Switzerland. This article is an open access article distributed under the terms and conditions of the Creative Commons Attribution (CC BY) license (https:// creativecommons.org/licenses/by/ $4.0 /)$.

\begin{abstract}
In this paper, we consider Bell-based Stirling polynomials of the second kind and derive some useful relations and properties including some summation formulas related to the Bell polynomials and Stirling numbers of the second kind. Then, we introduce Bell-based Bernoulli polynomials of order $\alpha$ and investigate multifarious correlations and formulas including some summation formulas and derivative properties. Also, we acquire diverse implicit summation formulas and symmetric identities for Bell-based Bernoulli polynomials of order $\alpha$. Moreover, we attain several interesting formulas of Bell-based Bernoulli polynomials of order $\alpha$ arising from umbral calculus.
\end{abstract}

Keywords: Bernoulli polynomials; bell polynomials; mixed-type polynomials; stirling numbers of the second kind; umbral calculus; summation formulas; derivative properties

\section{Introduction}

Special polynomials and numbers possess much importance in multifarious areas of sciences such as physics, mathematics, applied sciences, engineering and other related research fields covering differential equations, number theory, functional analysis, quantum mechanics, mathematical analysis, mathematical physics and so on, $c f$. [1-25] and see also each of the references cited therein. For example; Bernoulli polynomials and numbers are closely related to the Riemann zeta function, which possesses a connection with the distribution of prime numbers, $c f$. [22,24]. Some of the most significant polynomials in the theory of special polynomials are the Bell, Euler, Bernoulli, Hermite, and Genocchi polynomials. Recently, the aforesaid polynomials and their diverse generalizations have been densely considered and investigated by many physicists and mathematicians, $c f$. $[1-22,26]$ and see also the references cited therein.

In recent years, properties of special polynomials arising from umbral calculus have been studied and examined by several mathematicians. For instance, Dere et al. [7] considered Hermite base Bernoulli type polynomials and, by applying the umbral algebra to these polynomials, gave new identities for the Bernoulli polynomials of higher order, the Hermite polynomials and the Stirling numbers of the second kind. Kim et al. [11] acquired several new formulas for the Bernulli polynomials based upon the theory of the umbral calculus. Kim et al. [12] derived some identities of Bernoulli, Euler and Abel polynomials arising from umbral calculus. Kim et al. [14] studied partially degenerate Bell numbers and polynomials by using umbral calculus and derived some new identities. Kim et al. [16] investigated some properties and new identities for the degenerate ordered Bell polynomials associated with special polynomials derived from umbral calculus.

In this paper, we consider Bell-based Stirling polynomials of the second kind and derive some useful relations and properties including some summation formulas related to the Bell polynomials and Stirling numbers of the second kind. Then, we introduce Bell-based Bernoulli polynomials of order $\alpha$ and investigate multifarious correlations 
and formulas including some summation formulas and derivative properties. Also, we acquire diverse implicit summation formulas and symmetric identities for Bell-based Bernoulli polynomials of order $\alpha$. Moreover, we analyze some special cases of the results. Furthermore, we attain several interesting formulas of Bell-based Bernoulli polynomials of order $\alpha$ arising from umbral calculus to have alternative ways of deriving our results.

\section{Preliminaries}

Throughout this paper, the familiar symbols $\mathbb{C}, \mathbb{R}, \mathbb{Z}, \mathbb{N}$ and $\mathbb{N}_{0}$ refer to the set of all complex numbers, the set of all real numbers, the set of all integers, the set of all natural numbers and the set of all non-negative integers, respectively.

The Stirling polynomials $S_{2}(n, k: x)$ and numbers $S_{2}(n, k)$ of the second kind are given by the following exponential generating functions ( $c f .[3,8,13,15,26])$ :

$$
\sum_{n=0}^{\infty} S_{2}(n, k: x) \frac{t^{n}}{n !}=\frac{\left(e^{t}-1\right)^{k}}{k !} e^{t x} \text { and } \sum_{n=0}^{\infty} S_{2}(n, k) \frac{t^{n}}{n !}=\frac{\left(e^{t}-1\right)^{k}}{k !} .
$$

In combinatorics, Stirling numbers of the second kind $S_{2}(n, k)$ counts the number of ways in which $n$ distinguishable objects can be partitioned into $k$ indistinguishable subsets when each subset has to contain at least one object. The Stirling numbers of the second kind can also be derived by the following recurrence relation for $\zeta \in \mathbb{N}_{0}(c f .[3,8,13,15,26])$ :

$$
x^{n}=\sum_{k=0}^{n} S_{2}(n, k)(x)_{k}
$$

where $(x)_{n}=x(x-1)(x-2) \cdots(x-(n-1))$ for $n \in \mathbb{N}$ with $(x)_{0}=1$ (see $\left.[4,18,19]\right)$.

For each integer $k \in \mathbb{N}_{0}, S_{k}(n)=\sum_{l=0}^{n} l^{k}$ is named the sum of integer powers. The exponential generating function of $S_{k}(n)$ is as follows (cf. [20]):

$$
\sum_{k=0}^{\infty} S_{k}(n) \frac{t^{k}}{k !}=\frac{e^{(n+1) t}-1}{e^{t}-1}
$$

The bivariate Bell polynomials are defined as follows:

$$
\sum_{n=0}^{\infty} B e l_{n}(x ; y) \frac{t^{n}}{n !}=e^{y\left(e^{t}-1\right)} e^{x t}
$$

When $x=0, \operatorname{Bel}_{n}(0 ; y):=\operatorname{Bel}_{n}(y)$ is called the classical Bell polynomials (also called exponential polynomials) given by means of the following generating function $(c f .[3,4,9,26])$ :

$$
\sum_{n=0}^{\infty} B e l_{n}(y) \frac{t^{n}}{n !}=e^{y\left(e^{t}-1\right)}
$$

The Bell numbers $B e l_{n}$ are attained by taking $y=1$ in (5), that is $B e l_{n}(0 ; 1)=B e l_{n}(1):=$ $B e l_{n}$ and are given by the following exponential generating function $(c f .[3,4,9,26])$ :

$$
\sum_{n=0}^{\infty} B e l_{n} \frac{t^{n}}{n !}=e^{\left(e^{t}-1\right)}
$$

The Bell polynomials considered by Bell [26] appear as a standard mathematical tool and arise in combinatorial analysis. Since the first consideration of the Bell polynomials, these polynomials have been intensely investigated and studied by several mathematicians, $c f$. $[2,3,8,12-16,22,26]$ and see also the references cited therein. 
The usual Bell polynomials and Stirling numbers of the second kind satisfy the following relation ( $c f$. [9])

$$
\operatorname{Bel}_{n}(y)=\sum_{m=0}^{n} S_{2}(n, m) y^{m}
$$

The Bernoulli polynomials $B_{n}^{(\alpha)}(x)$ of order $\alpha$ are defined as follows $(c f .[1,7,11,12,18,21])$ :

$$
\sum_{n=0}^{\infty} B_{n}^{(\alpha)}(x) \frac{t^{n}}{n !}=\left(\frac{t}{e^{t}-1}\right)^{\alpha} e^{x t} \quad(|t|<2 \pi) .
$$

Setting $x=0$ in (8), we get $B_{n}^{(\alpha)}(0):=B_{n}^{(\alpha)}$ known as the Bernoulli numbers of order $\alpha$. We also note that when $\alpha=1$ in (8), the polynomials $B_{n}^{(\alpha)}(x)$ and numbers $B_{n}^{(\alpha)}$ reduce to the classical Bernoulli polynomials $B_{n}(x)$ and numbers $B_{n}$.

\section{Bell-Based Stirling Polynomials of the Second Kind}

In this section, we introduce the Bell-based Stirling polynomials of the second kind and analyze their elementary properties and relations. follows.

Here is the definition of the Bell-based Stirling polynomials of the second kind as

Definition 1. The Bell-based Stirling polynomials of the second kind are introduced by the following generating function:

$$
\sum_{n=0}^{\infty} B_{B e l} S_{2}(n, k: x, y) \frac{t^{n}}{n !}=\frac{\left(e^{t}-1\right)^{k}}{k !} e^{x t+y\left(e^{t}-1\right)}
$$

Diverse special circumstances of ${ }_{B e l} S_{2}(n, k: x, y)$ are discussed below:

Remark 1. Replacing $x=0$ in (9), we acquire Bell-Stirling polynomials ${ }_{B e l} S_{2}(n, k: y)$ of the second kind, which are also a new generalization of the usual Stirling numbers of the second kind in (1), as follows:

$$
\sum_{n=0}^{\infty} B_{B e l} S_{2}(n, k: y) \frac{t^{n}}{n !}=\frac{\left(e^{t}-1\right)^{k}}{k !} e^{y\left(e^{t}-1\right)}
$$

Remark 2. Substituting $y=0$ in (9), we get the Stirling polynomials of the second kind given by (1), cf. $[11,21,22]$.

Remark 3. Upon setting $x=y=0$ in (9), the Bell-based Stirling polynomials of the second kind reduce to the classical Stirling numbers of the second kind $S_{2}(n, k)$ by $(1), c f .[9,22,24]$.

We now ready to derive some properties of the ${ }_{B e l} S_{2}(n, k: x, y)$.

Theorem 1. The following correlation

$$
{ }_{B e l} S_{2}(n, k: x, y)=\sum_{u=0}^{n}\left(\begin{array}{l}
n \\
u
\end{array}\right) S_{2}(u, k) \operatorname{Bel}_{n-u}(x ; y)
$$

holds for non-negative integer $n$. 
Proof. This theorem is proved by (1), (4), and (9), as follows:

$$
\begin{aligned}
\sum_{n=0}^{\infty} B e l S_{2}(n, k: x, y) \frac{t^{n}}{n !} & =\frac{\left(e^{t}-1\right)^{k}}{k !} e^{x t+y\left(e^{t}-1\right)} \\
& =\sum_{n=k}^{\infty} S_{2}(n, k) \frac{t^{n}}{n !} \sum_{n=0}^{\infty} B e l_{n}(x ; y) \frac{t^{n}}{n !} \\
& =\sum_{n=0}^{\infty} \sum_{u=0}^{n}\left(\begin{array}{l}
n \\
u
\end{array}\right) S_{2}(u, k) B e l_{n-u}(x ; y) \frac{t^{n}}{n !}
\end{aligned}
$$

which provides the desired result (11).

Remark 4. Theorem 1 gives the following formula including the Stirling numbers of the second kind, the Bell-Stirling polynomials of the second kind, and Bell polynomials:

$$
{ }_{B e l} S_{2}(n, k: y)=\sum_{u=0}^{n}\left(\begin{array}{l}
n \\
u
\end{array}\right) S_{2}(u, k) B e l_{n-u}(y)
$$

Theorem 2. The following relations

$$
{ }_{B e l} S_{2}(n, k: x, y)=\sum_{l=0}^{n}\left(\begin{array}{c}
n \\
l
\end{array}\right){ }_{B e l} S_{2}(l, k: y) x^{n-l}
$$

and

$$
{ }_{B e l} S_{2}(n, k: x, y)=\sum_{l=0}^{n}\left(\begin{array}{l}
n \\
l
\end{array}\right) S_{2}(l, k: x) \operatorname{Bel}_{n-l}(y)
$$

hold for non-negative integers $n$ and $k$ with $n \geq k$.

Proof. The proofs are similar to Theorem 1.

Theorem 3. The following summation formulae for Bell-based Stirling polynomials of the second kind

$$
{ }_{B e l} S_{2}\left(n, k: x_{1}+x_{2}, y\right)=\sum_{u=0}^{n}\left(\begin{array}{l}
n \\
u
\end{array}\right){ }_{B e l} S_{2}\left(u, k: x_{1}, y\right) x_{2}^{n-u}
$$

and

$$
{ }_{B e l} S_{2}\left(n, k: x, y_{1}+y_{2}\right)=\sum_{u=0}^{n}\left(\begin{array}{l}
n \\
u
\end{array}\right){ }_{B e l} S_{2}\left(u, k: x, y_{1}\right) \operatorname{Bel}_{n-u}\left(y_{2}\right)
$$

hold for non-negative integers $n$ and $k$ with $n \geq k$.

Proof. Using the following equalities

$$
e^{\left(x_{1}+x_{2}\right) t+y\left(e^{t}-1\right)}=e^{x_{1} t+y\left(e^{t}-1\right)} e^{x_{2} t} \text { and } e^{x t+\left(y_{1}+y_{2}\right)\left(e^{t}-1\right)}=e^{x t+y_{1}\left(e^{t}-1\right)} e^{y_{2}\left(e^{t}-1\right)},
$$

the proofs are similar to Theorem 1. So, we omit them.

Theorem 4. The following relation

$$
{ }_{B e l} S_{2}\left(n, k_{1}+k_{2}: x, y\right)=\frac{k_{1} ! k_{2} !}{\left(k_{1}+k_{2}\right) !} \sum_{u=0}^{n}\left(\begin{array}{l}
n \\
u
\end{array}\right){ }_{B e l} S_{2}\left(u, k_{1}: x, y\right) S_{2}\left(n-u, k_{2}\right)
$$

is valid for non-negative integer $n$. 
Proof. In view of (1) and (9), we have

$$
\begin{gathered}
\sum_{n=0}^{\infty}{ }_{B e l} S_{2}\left(n, k_{1}+k_{2}: x, y\right) \frac{t^{n}}{n !}=\frac{\left(e^{t}-1\right)^{k_{1}+k_{2}}}{\left(k_{1}+k_{2}\right) !} e^{x t+y\left(e^{t}-1\right)} \\
=\frac{k_{1} ! k_{2} !}{\left(k_{1}+k_{2}\right) !} \frac{\left(e^{t}-1\right)^{k_{1}}}{k_{1}} e^{x t+y\left(e^{t}-1\right) \frac{\left(e^{t}-1\right)^{k_{2}}}{k_{2} !}} \\
=\frac{k_{1} ! k_{2} !}{\left(k_{1}+k_{2}\right) !} \sum_{n=0}^{\infty} \sum_{u=0}^{n}\left(\begin{array}{l}
n \\
u
\end{array}\right){ }_{B e l} S_{2}\left(u, k_{1}: x, y\right) S_{2}\left(n-u, k_{2}\right) \frac{t^{n}}{n !},
\end{gathered}
$$

which gives the asserted result (16).

Theorem 5. The following relation

$$
S_{2}(n, k)=\sum_{u=0}^{n}\left(\begin{array}{l}
n \\
u
\end{array}\right){ }_{B e l} S_{2}(u, k: x, y) \operatorname{Bel}_{n-u}(-x ;-y)
$$

holds for non-negative integer $n$.

Proof. Utilizing the following equality

$$
\frac{\left(e^{t}-1\right)^{k}}{k !}=\frac{\left(e^{t}-1\right)^{k}}{k !} e^{x t+y\left(e^{t}-1\right)} e^{-x t-y\left(e^{t}-1\right)},
$$

it is similar to Theorem 1 . So, we omit the proof.

Remark 5. Theorem 5 gives the following formula including the Stirling numbers of the second kind, the Bell-Stirling polynomials of the second kind, and Bell polynomials:

$$
S_{2}(n, k)=\sum_{u=0}^{n}\left(\begin{array}{l}
n \\
u
\end{array}\right){ }_{B e l} S_{2}(u, k:-y) \operatorname{Bel}_{n-u}(y) .
$$

\section{Bell-Based Bernoulli Polynomials and Numbers of Order $\alpha$}

In this section, we introduce Bell-based Bernoulli polynomials of order $\alpha$ and investigate multifarious correlations and formulas including summation formulas, derivation rules, and correlations with the Bell-based Stirling numbers of the second kind.

We now introduce Bell-based Bernoulli polynomials of order $\alpha$ as follows.

Definition 2. The Bell-based Bernoulli polynomials of order $\alpha$ are defined by the following exponential generating function:

$$
\sum_{n=0}^{\infty} B_{e l} B_{n}^{(\alpha)}(x ; y) \frac{t^{n}}{n !}=\left(\frac{t}{e^{t}-1}\right)^{\alpha} e^{x t+y\left(e^{t}-1\right)} .
$$
below.

Some special cases of the Bell-based Bernoulli polynomials of order $\alpha$ are analyzed

Remark 6. In the special case $x=0$ in (18), we acquire Bell-Bernoulli polynomials $B_{B e l} B_{n}^{(\alpha)}(y)$ of order $\alpha$, which are also new extensions of the Bernoulli numbers of order $\alpha$ in (8), as follows:

$$
\sum_{n=0}^{\infty} B e l B_{n}^{(\alpha)}(y) \frac{t^{n}}{n !}=\left(\frac{t}{e^{t}-1}\right)^{\alpha} e^{y\left(e^{t}-1\right)}
$$

Remark 7. Upon letting $y=0$ in (18), the Bell-based Bernoulli polynomials ${ }_{B e l} B_{n}^{(\alpha)}(x ; y)$ of order $\alpha$ reduce to the familiar Bernoulli polynomials $B_{n}^{(\alpha)}(x)$ of order $\alpha$ in (8). 
Remark 8. When $y=0$ and $\alpha=1$, the polynomials ${ }_{B e l} B_{n}^{(\alpha)}(x ; y)$ reduce to the usual Bernoulli polynomials $B_{n}(x)$.

We also note that

$$
{ }_{B e l} B_{n}^{(1)}(x ; y):={ }_{B e l} B_{n}(x ; y),
$$

which we call the Bell-based Bernoulli polynomials.

We now perform to derive some properties of the Bell-based Bernoulli polynomials of order $\alpha$ and we first provide the following theorem.

Theorem 6. Each of the following summation formulae

$$
\begin{gathered}
{ }_{B e l} B_{n}^{(\alpha)}(x ; y)=\sum_{k=0}^{n}\left(\begin{array}{l}
n \\
k
\end{array}\right) B_{k}^{(\alpha)} \operatorname{Bel}_{n-k}(x ; y) \\
B_{e l} B_{n}^{(\alpha)}(x ; y)=\sum_{k=0}^{n}\left(\begin{array}{l}
n \\
k
\end{array}\right) B_{k}^{(\alpha)}(x) B_{e l} l_{n-k}(y) \\
{ }_{B e l} B_{n}^{(\alpha)}(x ; y)=\sum_{k=0}^{n}\left(\begin{array}{l}
n \\
k
\end{array}\right){ }_{B e l} B_{k}^{(\alpha)}(y) x^{n-k}
\end{gathered}
$$

hold for $n \in \mathbb{N}_{0}$.

Proof. They are similar to Theorem 1. So, we omit them.

We provide an implicit summation formula for the Bell-based Bernoulli polynomials by the following theorem.

Theorem 7. The following relationship

$$
\mathrm{Bel}_{n}^{\left(\alpha_{1}+\alpha_{2}\right)}\left(x_{1}+x_{2} ; y_{1}+y_{2}\right)=\sum_{k=0}^{n}\left(\begin{array}{l}
n \\
k
\end{array}\right){ }_{B e l} B_{k}^{\left(\alpha_{1}\right)}\left(x_{1} ; y_{1}\right)_{B e l} B_{n-k}^{\left(\alpha_{2}\right)}\left(x_{2} ; y_{2}\right)
$$

is valid for $n \in \mathbb{N}_{0}$.

Proof. Using the following equality

$$
\frac{t^{\alpha_{1}+\alpha_{2}}}{\left(e^{t}-1\right)^{\alpha_{1}+\alpha_{2}}} e^{\left(x_{1}+x_{2}\right) t+\left(y_{1}+y_{2}\right)\left(e^{t}-1\right)}=\frac{t^{\alpha_{1}}}{\left(e^{t}-1\right)^{\alpha_{1}}} e^{x_{1} t+y_{1}\left(e^{t}-1\right)} \frac{t^{\alpha_{2}}}{\left(e^{t}-1\right)^{\alpha_{2}}} e^{x_{2} t+y_{2}\left(e^{t}-1\right)},
$$

the proof is similar to Theorem 1. So, we omit it.

One of the special cases of Theorem 7 is given, for every $n \in \mathbb{N}_{0}$, by

$$
{ }_{B e l} B_{n}^{(\alpha)}(x+1 ; y)=\sum_{k=0}^{n}\left(\begin{array}{l}
n \\
k
\end{array}\right){ }_{B e l} B_{k}^{(\alpha)}(x ; y)
$$

which is a generalization of the well-known formula for usual Bernoulli polynomials given by

$$
B_{n}(x+1)=\sum_{k=0}^{n}\left(\begin{array}{l}
n \\
k
\end{array}\right) B_{k}(x) \quad(c f .[11])
$$

We now provide derivative operator properties for the polynomials ${ }_{B e l} B_{n}^{(\alpha)}(x ; y)$ as follows. 
Theorem 8. The difference operator formulas for the Bell-based Bernoulli polynomials

$$
\frac{\partial}{\partial x} B e l B_{n}^{(\alpha)}(x ; y)=n_{B e l} B_{n-1}^{(\alpha)}(x ; y)
$$

and

$$
\frac{\partial}{\partial y} B_{e l} B_{n}^{(\alpha)}(x ; y)=n_{B e l} B_{n-1}^{(\alpha-1)}(x ; y)
$$

hold for $n \in \mathbb{N}$.

Proof. Based on the following derivative properties

$$
\frac{\partial}{\partial x} e^{x t+y\left(e^{t}-1\right)}=t e^{x t+y\left(e^{t}-1\right)} \text { and } \frac{\partial}{\partial y} e^{x t+y\left(e^{t}-1\right)}=\left(e^{t}-1\right) e^{x t+y\left(e^{t}-1\right)},
$$

the proof is completed.

A recurrence relation for the Bell-based Bernoulli polynomials is given by the following theorem.

Theorem 9. The following summation formula

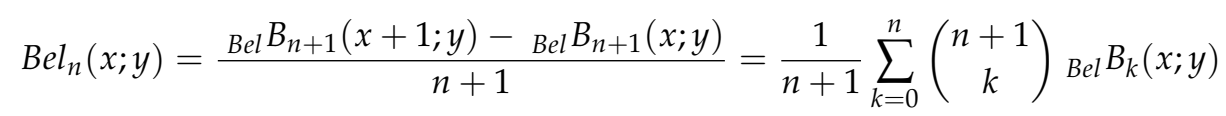

holds for $n \in \mathbb{N}_{0}$.

Proof. By means of Definition 2, based on the following equality

$$
e^{x t+y\left(e^{t}-1\right)}=\frac{e^{t}-1}{t} \sum_{n=0}^{\infty} B e l B_{n}(x ; y) \frac{t^{n}}{n !},
$$

the proof is done.

Remark 9. The result (27) is an extension of the well-known formula for Bernoulli polynomials given by (cf. [22,23])

$$
x^{n}=\frac{B_{n+1}(x+1)-B_{n+1}(x)}{n+1}
$$

An explicit formula for the Bell-based Bernoulli polynomials is given by the following theorem.

Theorem 10. The following explicit formula

$$
{ }_{B e l} B_{n}(x ; y)=\sum_{k=0}^{\infty} \sum_{l=0}^{k-1} y^{k}\left(\begin{array}{c}
k-1 \\
l
\end{array}\right)(-1)^{k-l-1} \frac{(l+x)^{n+1}}{n+1}
$$

holds for $n \in \mathbb{N}_{0}$. 
Proof. By means of Definition 2, based on the following equality

$$
\begin{aligned}
\sum_{n=0}^{\infty} B e l B_{n}(x ; y) \frac{t^{n}}{n !} & =\frac{t e^{x t}}{e^{t}-1} e^{y\left(e^{t}-1\right)}=t e^{x t} \sum_{k=0}^{\infty} y^{k}\left(e^{t}-1\right)^{k-1} \\
& =t \sum_{k=0}^{\infty} \sum_{l=0}^{k-1} y^{k}\left(\begin{array}{c}
k-1 \\
l
\end{array}\right)(-1)^{k-l-1} e^{(l+x) t} \\
& =\sum_{n=0}^{\infty} \sum_{k=0}^{\infty} \sum_{l=0}^{k-1} y^{k}\left(\begin{array}{c}
k-1 \\
l
\end{array}\right)(-1)^{k-l-1}(l+x)^{n} \frac{t^{n-1}}{n !},
\end{aligned}
$$

which gives the asserted result.

We give the following theorem.

Theorem 11. The following formula including the Bell-based Bernoulli polynomials of higher-order and Stirling numbers of the second kind

$$
\operatorname{Bel}_{n}(x ; y)=\frac{n ! k !}{(n+k) !} \sum_{l=0}^{n+k}\left(\begin{array}{c}
n+k \\
l
\end{array}\right){ }_{B e l} B_{l}^{(k)}(x ; y) S_{2}(n+k-l, m)
$$

is valid for $n \in \mathbb{N}_{0}$ and $k \in \mathbb{N}$.

Proof. By means of Definition 2, based on the following equality

$$
e^{x t+y\left(e^{t}-1\right)}=k ! t^{-k} \frac{\left(e^{t}-1\right)^{k}}{k !} \sum_{n=0}^{\infty} B e l B_{n}^{(k)}(x ; y) \frac{t^{n}}{n !},
$$

the proof is completed.

Here, we present the following theorem including the Bell-based Bernoulli polynomials and the Stirling polynomials of the second kind.

Theorem 12. The following correlation

$$
{ }_{B e l} B_{n}^{(\alpha)}(x ; y)=\sum_{l=0}^{n} \sum_{k=0}^{\infty}\left(\begin{array}{l}
n \\
l
\end{array}\right)(x)_{k} S_{2}(l, k)_{B e l} B_{n-l}^{(\alpha)}(y)
$$

holds for non-negative integers $n$.

Proof. By means of Definition 2 and, using (1) and (19), we obtain

$$
\begin{aligned}
\sum_{n=0}^{\infty} B e l B_{n}^{(\alpha)}(x ; y) \frac{t^{n}}{n !} & =\frac{t^{\alpha}}{\left(e^{t}-1\right)^{\alpha}} e^{y\left(e^{t}-1\right)}\left(e^{t}-1+1\right)^{x} \\
& =\frac{t^{\alpha}}{\left(e^{t}-1\right)^{\alpha}} e^{y\left(e^{t}-1\right)} \sum_{k=0}^{\infty}(x)_{k} \frac{\left(e^{t}-1\right)^{k}}{k !} \\
& =\sum_{n=0}^{\infty} \sum_{l=0}^{n} \sum_{k=0}^{\infty}\left(\begin{array}{l}
n \\
l
\end{array}\right)(x)_{k} S_{2}(l, k)_{B e l} B_{n-l}^{(\alpha)}(y) \frac{t^{n}}{n !},
\end{aligned}
$$

which gives the asserted result (29).

A correlation including the Bell-based Bernoulli polynomials of order $\alpha$ and the Bellbased Stirling polynomials of the second kind is stated below. 
Theorem 13. The following summation formula

$$
\operatorname{Bel}_{n}\left(x_{1}+x_{2} ; y_{1}+y_{2}\right)=\frac{n ! k !}{(n+k) !} \sum_{l=0}^{n+k}\left(\begin{array}{c}
n+k \\
l
\end{array}\right){ }_{B e l} B_{l}^{(k)}\left(x_{2} ; y_{2}\right){ }_{B e l} S_{2}\left(n+k-l, k: x_{1}, y_{1}\right)
$$

holds for non-negative integers $k$ and $n$ with $n \geq k$.

Proof. By (4) and (9), we have

$$
\sum_{n=0}^{\infty} B e l S_{2}\left(n, k: x_{1}, y_{1}\right) \frac{t^{n}}{n !} \sum_{n=0}^{\infty} B e l B_{n}^{(k)}\left(x_{2} ; y_{2}\right) \frac{t^{n}}{n !}=\frac{t^{k}}{k !} e^{\left(x_{1}+x_{2}\right) t+\left(y_{1}+y_{2}\right)\left(e^{t}-1\right)},
$$

which implies the claimed result (30).

Recently, implicit summation formulas and symmetric identities for special polynomials have been studied by some mathematicians, $c f$. $[8,20]$ and see the references cited therein. Now, we investigate some implicit summation formula and symmetric identities for Bell-based Bernoulli polynomials of order $\alpha$.

We note that the following series manipulation formulas hold (cf. [20,24]):

$$
\sum_{N=0}^{\infty} f(N) \frac{(x+y)^{N}}{N !}=\sum_{n, m=0}^{\infty} f(n+m) \frac{x^{n}}{n !} \frac{y^{m}}{m !}
$$

and

$$
\sum_{k, l=0}^{\infty} A(l, k)=\sum_{k=0}^{\infty} \sum_{l=0}^{k} A(l, k-l) .
$$

We give the following theorem.

Theorem 14. The following implicit summation formula

$$
{ }_{B e l} B_{k+l}^{(\alpha)}(x ; y)=\sum_{n, m=0}^{k, l}\left(\begin{array}{c}
k \\
n
\end{array}\right)\left(\begin{array}{c}
l \\
m
\end{array}\right)(x-z)^{n+m}{ }_{B e l} B_{k+l-n-m}^{(\alpha)}(z ; y)
$$

holds.

Proof. Upon setting $t$ by $t+u$ in (18), we derive

$$
\left(\frac{t+u}{e^{t+u}-1}\right)^{\alpha} e^{y\left(e^{t+u}-1\right)}=e^{-z(t+u)} \sum_{k, l=0}^{\infty} B e l B_{k+l}^{(\alpha)}(z ; y) \frac{t^{k}}{k !} \frac{u^{l}}{l !} .
$$

Again, replacing $z$ by $x$ in the last equation, and using (31), we get

$$
e^{-x(t+u)} \sum_{k, l=0}^{\infty} B e l B_{k+l}^{(\alpha)}(x ; y) \frac{t^{k}}{k !} \frac{u^{l}}{l !}=\left(\frac{t+u}{e^{t+u}-1}\right)^{\alpha} e^{y\left(e^{t+u}-1\right)}
$$

By the last two equations, we obtain

$$
\sum_{k, l=0}^{\infty} B e l B_{k+l}^{(\alpha)}(x ; y) \frac{t^{k}}{k !} \frac{u^{l}}{l !}=e^{(x-z)(t+u)} \sum_{k, l=0}^{\infty} B e l B_{k+l}^{(\alpha)}(z ; y) \frac{t^{k}}{k !} \frac{u^{l}}{l !}
$$

which yields

$$
\sum_{k, l=0}^{\infty} B e l B_{k+l}^{(\alpha)}(x ; y) \frac{t^{k}}{k !} \frac{u^{l}}{l !}=\sum_{n, m=0}^{\infty}(x-z)^{n+m} \frac{t^{n}}{n !} \frac{u^{m}}{m !} \sum_{k, l=0}^{\infty} B e l B_{k+l}^{(\alpha)}(z ; y) \frac{t^{k}}{k !} \frac{u^{l}}{l !} .
$$


Utilizing (32), we acquire

$$
\sum_{k, l=0}^{\infty} B e l B_{k+l}^{(\alpha)}(x ; y) \frac{t^{k}}{k !} \frac{u^{l}}{l !}=\sum_{k, l=0}^{\infty} \sum_{n, m=0}^{k, l} \frac{(x-z)^{n+m}{ }_{B e l} B_{k+l-n-m}^{(\alpha)}(z ; y)}{n ! m !(k-l) !(l-m) !} t^{k} u^{l},
$$

which implies the asserted result (33).

Corollary 1. Letting $k=0$ in (33), the following implicit summation formula holds:

$$
{ }_{B e l} B_{l}^{(\alpha)}(x ; y)=\sum_{m=0}^{l}\left(\begin{array}{c}
l \\
m
\end{array}\right)(x-z)^{m}{ }_{B e l} B_{l-m}^{(\alpha)}(z ; y) .
$$

Corollary 2. Upon setting $k=0$ and replacing $x$ by $x+z$ in (33), we attain

$$
{ }_{B e l} B_{l}^{(\alpha)}(x+z ; y)=\sum_{m=0}^{l}\left(\begin{array}{c}
l \\
m
\end{array}\right) x_{B e l}^{m} B_{l-m}^{(\alpha)}(z ; y) .
$$

Now, we give the following theorem.

Theorem 15. The following symmetric identity

$$
\sum_{k=0}^{n}\left(\begin{array}{l}
n \\
k
\end{array}\right) B_{B e l} B_{n-k}^{(\alpha)}(b x ; y)_{B e l} B_{k}^{(\alpha)}(a x ; y) a^{n-k} b^{k}=\sum_{k=0}^{n}\left(\begin{array}{l}
n \\
k
\end{array}\right) B e l B_{k}^{(\alpha)}(b x ; y)_{B e l} B_{n-k}^{(\alpha)}(a x ; y) a^{k} b^{n-k}
$$

holds for $a, b \in \mathbb{R}$ and $n \geq 0$.

Proof. Let

$$
\mathrm{Y}=\left(\frac{t^{2}}{\left(e^{a t}-1\right)\left(e^{b t}-1\right)}\right)^{\alpha} e^{2 a b x t+y\left(e^{a t}-1\right)+y\left(e^{b t}-1\right)} .
$$

Then, the expression for $\mathrm{Y}$ is symmetric in $a$ and $b$, and we derive the following two expansions of $\mathrm{Y}$ :

$$
\begin{aligned}
\mathrm{Y} & =\sum_{n=0}^{\infty}{ }_{B e l} B_{n}^{(\alpha)}(b x ; y) \frac{(a t)^{n}}{n !} \sum_{n=0}^{\infty}{ }_{B e l} B_{n}^{(\alpha)}(a x ; y) \frac{(b t)^{n}}{n !} \\
& =\sum_{n=0}^{\infty} \sum_{k=0}^{n}\left(\begin{array}{l}
n \\
k
\end{array}\right){ }_{B e l} B_{n-k}^{(\alpha)}(b x ; y)_{B e l} B_{k}^{(\alpha)}(a x ; y) a^{n-k} b^{k} \frac{t^{n}}{n !}
\end{aligned}
$$

and, similarly,

$$
\mathrm{Y}=\sum_{n=0}^{\infty} \sum_{k=0}^{n}\left(\begin{array}{l}
n \\
k
\end{array}\right){ }_{B e l} B_{k}^{(\alpha)}(b x ; y){ }_{B e l} B_{n-k}^{(\alpha)}(a x ; y) a^{k} b^{n-k} \frac{t^{n}}{n !},
$$

which gives the desired result (34).

Here is another symmetric identity for ${ }_{B e l} B_{n}^{(\alpha)}(x ; y)$ as follows.

Theorem 16. Let $a, b \in \mathbb{R}$ and $n \geq 0$. Then the following identity holds:

$$
\begin{aligned}
& \sum_{k=0}^{n} \sum_{i=0}^{b-1} \sum_{j=0}^{a-1}\left(\begin{array}{l}
n \\
k
\end{array}\right){ }_{B e l} B_{k}^{(\alpha)}\left(i+\frac{b}{a} j+b x_{1} ; y\right){ }_{B e l} B_{n-k}^{(\alpha)}\left(a x_{2} ; y\right) a^{k} b^{n-k} \\
= & \sum_{k=0}^{n} \sum_{i=0}^{b-1} \sum_{j=0}^{a-1}\left(\begin{array}{l}
n \\
k
\end{array}\right){ }_{B e l} B_{k}^{(\alpha)}\left(\frac{a}{b} i+j+a x_{2} ; y\right){ }_{B e l} B_{n-k}^{(\alpha)}\left(b x_{1} ; y\right) b^{k} a^{n-k} .
\end{aligned}
$$


Proof. Let

$$
\begin{aligned}
\Psi & =\frac{(a t)^{\alpha}(b t)^{\alpha}}{\left(e^{a t}-1\right)^{\alpha+1}\left(e^{b t}-1\right)^{\alpha+1}}\left(e^{a b t}-1\right)^{2} e^{a b\left(x_{1}+x_{2}\right) t+y\left(e^{a t}-1\right)+x\left(e^{b t}-1\right)} \\
& =\left(\frac{a t}{e^{a t}-1}\right)^{\alpha}\left(\frac{e^{a b t}-1}{e^{a t}-1}\right) e^{a b x_{1} t+y\left(e^{a t}-1\right)}\left(\frac{b t}{e^{b t}-1}\right)^{\alpha}\left(\frac{e^{a b t}-1}{e^{b t}-1}\right) e^{a b x_{2} t+y\left(e^{b t}-1\right)}
\end{aligned}
$$

By (18), the formula (36) can be expanded as follows

$$
\begin{aligned}
\Psi & =\left(\frac{a t}{e^{a t}-1}\right)^{\alpha} e^{a b x_{1} t+y\left(e^{a t}-1\right)} \sum_{i=0}^{b-1} e^{a t i}\left(\frac{b t}{e^{b t}-1}\right)^{\alpha} e^{a b x_{2} t+y\left(e^{b t}-1\right)} \sum_{j=0}^{a-1} e^{b t j} \\
& =\sum_{i=0}^{b-1} \sum_{j=0}^{a-1}\left(\frac{a t}{e^{a t}-1}\right)^{\alpha} e^{\left(i+\frac{b}{a} j+b x_{1}\right) a t+y\left(e^{a t}-1\right)}\left(\frac{b t}{e^{b t}-1}\right)^{\alpha} e^{a b x_{2} t+y\left(e^{b t}-1\right)} \\
& =\sum_{n=0}^{\infty} \sum_{k=0}^{n} \sum_{i=0}^{b-1} \sum_{j=0}^{a-1}\left(\begin{array}{l}
n \\
k
\end{array}\right){ }_{B e l} B_{k}^{(\alpha)}\left(i+\frac{b}{a} j+b x_{1} ; y\right){ }_{B e l} B_{n-k}^{(\alpha)}\left(a x_{2} ; y\right) a^{k} b^{n-k} \frac{t^{n}}{n !}
\end{aligned}
$$

and similarly,

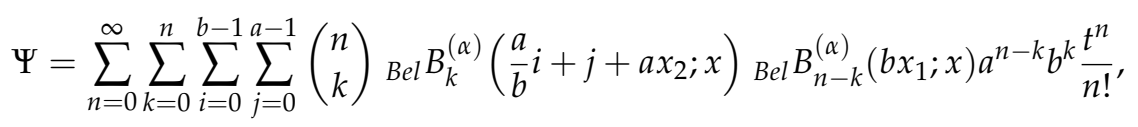

which means the claimed result (35).

Lastly, we provide the following symmetric identity.

Theorem 17. The following symmetric identity

$$
\begin{aligned}
& \sum_{l=0}^{n} \sum_{k=0}^{l}\left(\begin{array}{l}
n \\
l
\end{array}\right)\left(\begin{array}{l}
l \\
k
\end{array}\right) S_{n-l}(b-1)_{B e l} B_{k}^{(\alpha)}\left(b x_{1} ; y\right)_{B e l} B_{l-k}^{(\alpha+1)}\left(a x_{2} ; y\right) a^{n+k+1-l} b^{l-k} \\
= & \sum_{l=0}^{n} \sum_{k=0}^{l}\left(\begin{array}{l}
n \\
l
\end{array}\right)\left(\begin{array}{l}
l \\
k
\end{array}\right) S_{n-l}(a-1)_{B e l} B_{k}^{(\alpha)}\left(a x_{2} ; y\right)_{B e l} B_{l-k}^{(\alpha+1)}\left(b x_{1} ; y\right) b^{n+k+1-l} a^{l-k}
\end{aligned}
$$

holds for $a, b \in \mathbb{Z}$ and $n \geq 0$.

Proof. Let

$$
\Omega=\frac{(a t)^{\alpha+1}(b t)^{\alpha+1}}{\left(e^{a t}-1\right)^{\alpha+1}\left(e^{b t}-1\right)^{\alpha+1}}\left(e^{a b t}-1\right) e^{a b\left(x_{1}+x_{2}\right) t+y\left(e^{a t}-1\right)+y\left(e^{b t}-1\right)} .
$$

By (3) and (18), we observe that

$$
\begin{aligned}
\Omega & =a t\left(\frac{e^{a b t}-1}{e^{a t}-1}\right)\left(\frac{a t}{e^{a t}-1}\right)^{\alpha} e^{a b x_{1} t+y\left(e^{a t}-1\right)}\left(\frac{b t}{e^{b t}-1}\right)^{\alpha+1} e^{a b x_{2} t+y\left(e^{b t}-1\right)} \\
& =a t \sum_{n=0}^{\infty} S_{n}(b-1) \frac{(a t)^{n}}{n !} \sum_{n=0}^{\infty} B e l B_{n}^{(\alpha)}\left(b x_{1} ; y\right) \frac{(a t)^{n}}{n !} \sum_{n=0}^{\infty} B e l B_{n}^{(\alpha+1)}\left(a x_{2} ; y\right) \frac{(b t)^{n}}{n !} \\
& =\sum_{n=0}^{\infty} \sum_{l=0}^{n} \sum_{k=0}^{l}\left(\begin{array}{l}
n \\
l
\end{array}\right)\left(\begin{array}{l}
l \\
k
\end{array}\right) S_{n-l}(b-1)_{B e l} B_{k}^{(\alpha)}\left(b x_{1} ; y\right)_{B e l} B_{l-k}^{(\alpha+1)}\left(a x_{2} ; y\right) a^{n+k+1-l} b^{l-k} \frac{t^{n-1}}{n !}
\end{aligned}
$$


and also

$$
\Omega=\sum_{n=0}^{\infty} \sum_{l=0}^{n} \sum_{k=0}^{l}\left(\begin{array}{l}
n \\
l
\end{array}\right)\left(\begin{array}{l}
l \\
k
\end{array}\right) S_{n-l}(a-1)_{B e l} B_{k}^{(\alpha)}\left(a x_{2} ; y\right)_{B e l} B_{l-k}^{(\alpha+1)}\left(b x_{1} ; y\right) a^{l-k} b^{n+k+1-l} \frac{t^{n-1}}{n !},
$$

which imply the claimed result (37).

\section{Applications Arising from Umbral Calculus}

We now review briefly the concept of umbral calculus. For the properties of umbral calculus, we refer the reader to see the references $[1,4-6,9-11,13,15,18]$.

Let $\mathcal{F}$ be the set of all formal power series in the variable $t$ over $\mathbb{C}$ with

$$
\mathcal{F}=\left\{f \mid f(t)=\sum_{k=0}^{\infty} a_{k} \frac{t^{k}}{k !}, \quad\left(a_{k} \in \mathbb{C}\right)\right\} .
$$

Let $\mathbb{P}$ be the algebra of polynomials in the single variable $x$ over the field complex numbers and let $\mathbb{P}^{*}$ be the vector space of all linear functionals on $\mathbb{P}$. In the umbral calculus, $\langle L \mid p(x)\rangle$ means the action of a linear functional $L$ on the polynomial $p(x)$. This operator has a linear property on $\mathbb{P}^{*}$ given by

$$
\langle L+M \mid p(x)\rangle=\langle L \mid p(x)\rangle+\langle M \mid p(x)\rangle
$$

and

$$
\langle c L \mid p(x)\rangle=c\langle L \mid p(x)\rangle
$$

for any constant $c$ in $\mathbb{C}$.

The formal power series

$$
f(t)=\sum_{k=0}^{\infty} a_{k} \frac{t^{k}}{k !}
$$

defines a linear functional on $\mathbb{P}$ by setting

$$
\left\langle f(t) \mid x^{n}\right\rangle=a_{n} \quad(n \geq 0) .
$$

Taking $f(t)=t^{k}$ in (38) and (39) gives

$$
\left\langle t^{k} \mid x^{n}\right\rangle=n ! \delta_{n, k}, \quad(n, k \geq 0)
$$

where

$$
\delta_{n, k}=\left\{\begin{array}{ll}
1, & \text { if } n=k \\
0, & \text { if } n \neq k
\end{array} .\right.
$$

Actually, any linear functional $L$ in $\mathbb{P}^{*}$ has the form (38). That is, since

$$
f_{L}(t)=\sum_{k=0}^{\infty}\left\langle L \mid x^{k}\right\rangle \frac{t^{k}}{k !^{\prime}}
$$

we have

$$
\left\langle f_{L}(t) \mid x^{n}\right\rangle=\left\langle L \mid x^{n}\right\rangle,
$$

and so as linear functionals $L=f_{L}(t)$. Moreover, the map $L \rightarrow f_{L}(t)$ is a vector space isomorphism from $\mathbb{P}^{*}$ onto $\mathcal{F}$. Henceforth, $\mathcal{F}$ will denote both the algebra of formal power series in $t$ and the vector space of all linear functionals on $\mathbb{P}$, and so an element $f(t)$ of $\mathcal{F}$ will be thought of as both a formal power series and a linear functional. From (39), we have

$$
\left\langle e^{y t} \mid x^{n}\right\rangle=y^{n}
$$


and so

$$
\left\langle e^{y t} \mid p(x)\right\rangle=p(y) \quad(p(x) \in \mathbb{P}) .
$$

The order $o(f(t))$ of a power series $f(t)$ is the smallest integer $k$ for which the coefficient of $t^{k}$ does not vanish. If $o(f(t))=0$, then $f(t)$ is called an invertible series. A series $f(t)$ for which $o(f(t))=1$ will be called a delta series ( $c f$. [1,4-6,9-11,13,15,18]).

If $f_{1}(t), \ldots, f_{m}(t)$ are in $\mathcal{F}$, then

$$
\left\langle f_{1}(t) \ldots f_{m}(t) \mid x^{n}\right\rangle=\sum_{i_{1}+i_{2}+\ldots+i_{m}=n}\left(\begin{array}{c}
n \\
i_{1}, \ldots, i_{m}
\end{array}\right)\left\langle f_{1}(t) \mid x^{i_{1}}\right\rangle \ldots\left\langle f_{m}(t) \mid x^{i_{m}}\right\rangle,
$$

where

$$
\left(\begin{array}{c}
n \\
i_{1}, \cdots, i_{r}
\end{array}\right)=\frac{n !}{i_{1} ! \cdots i_{r} !} .
$$

We use the notation $t^{k}$ for the $k$-th derivative operator on $\mathbb{P}$ as follows:

$$
t^{k} x^{n}=\left\{\begin{array}{ll}
\frac{n !}{(n-k) !} x^{n-k}, & k \leq n \\
0, & k>n
\end{array} .\right.
$$

If $f(t)$ and $g(t)$ are in $\mathcal{F}$, then

$$
\langle f(t) g(t) \mid p(x)\rangle=\langle f(t) \mid g(t) p(x)\rangle=\langle g(t) \mid f(t) p(x)\rangle
$$

for all polynomials $p(x)$. Notice that for all $f(t)$ in $\mathcal{F}$, and for all polynomials $p(x)$,

$$
f(t)=\sum_{k=0}^{\infty}\left\langle f(t) \mid x^{k}\right\rangle \frac{t^{k}}{k !} \quad \text { and } \quad p(x)=\sum_{k=0}^{\infty}\left\langle t^{k} \mid p(x)\right\rangle \frac{x^{k}}{k !} .
$$

Using (43), we obtain

$$
p^{(k)}(x):=D^{k} p(x)=\sum_{l=k}^{\infty} \frac{\left\langle t^{l} \mid p(x)\right\rangle}{l !} x^{l-k} \prod_{s=1}^{k}(l-s+1)
$$

providing

$$
p^{(k)}(0)=\left\langle t^{k} \mid p(x)\right\rangle \quad \text { and } \quad\left\langle 1 \mid p^{(k)}(x)\right\rangle=p^{(k)}(0) .
$$

Thus, from (44), we note that

$$
t^{k} p(x)=p^{(k)}(x)
$$

Let $f(t) \in \mathcal{F}$ be a delta series and let $g(t) \in \mathcal{F}$ be an invertible series. Then there exists a unique sequence $s_{n}(x)$ of polynomials satisfying the following property:

$$
\left\langle g(t) f(t)^{k} \mid s_{n}(x)\right\rangle=n ! \delta_{n, k} \quad(n, k \geq 0),
$$

which is called an orthogonality condition for any Sheffer sequence, $c f$. [1,4-6,9-11,13,15,18,22].

The sequence $s_{n}(x)$ is called the Sheffer sequence for the pair of $(g(t), f(t))$, or this $s_{n}(x)$ is Sheffer for $(g(t), f(t))$, which is denoted by $s_{n}(x) \sim(g(t), f(t))$.

Let $s_{n}(x)$ be Sheffer for $(g(t), f(t))$. Then for any $h(t)$ in $\mathcal{F}$, and for any polynomial $p(x)$, we have

$$
h(t)=\sum_{k=0}^{\infty} \frac{\left\langle h(t) \mid s_{k}(x)\right\rangle}{k !} g(t) f(t)^{k}, \quad p(x)=\sum_{k=0}^{\infty} \frac{\left\langle g(t) f(t)^{k} \mid p(x)\right\rangle}{k !} s_{k}(x)
$$

and the sequence $s_{n}(x)$ is Sheffer for $(g(t), f(t))$ if and only if

$$
\frac{1}{g(\bar{f}(t))} e^{x \bar{f}(t)}=\sum_{n=0}^{\infty} s_{n}(x) \frac{t^{n}}{n !}
$$


for all $x$ in $\mathbb{C}$, where $\bar{f}(f(t))=f(\bar{f}(t))=t$.

An important property for the Sheffer sequence $s_{n}(x)$ having $(g(t), t)$ is the Appell sequence. It is also called Appell for $g(t)$ with the following consequence:

$$
s_{n}(x)=\frac{1}{g(t)} x^{n} \Leftrightarrow t s_{n}(x)=n s_{n-1}(x) .
$$

Further important property for Sheffer sequence $s_{n}(x)$ is as follows

$$
s_{n}(x) \text { is Appell for } g(t) \Leftrightarrow \frac{1}{g(t)} e^{x t}=\sum_{n=0}^{\infty} s_{n}(x) \frac{t^{n}}{n !}(x \in \mathbb{C}) .
$$

For further information about the properties of umbral theory, see [19] and cited references therein. Recently, several authors have studied Bernoulli polynomials, Euler polynomials with various generalizations under the theory of umbral calculus $[1,4-6,9-11,13,15,22]$.

Recall from (18) that

$$
\sum_{n=0}^{\infty} B e l B_{n}(x ; y) \frac{t^{n}}{n !}=\frac{t}{e^{t}-1} e^{x t+y\left(e^{t}-1\right)} .
$$

As $t$ approaches to 0 in (50) gives ${ }_{B e l} B_{0}(x ; y)=1$ that stands for $o\left(\frac{t}{e^{t}-1} e^{x t+y\left(e^{t}-1\right)}\right)=0$. It means that the generating function of Bell-based Bernoulli polynomials is invertible and thus can be used as an application of Sheffer sequence.

Now we list some properties of Bell-based Bernoulli polynomials arising from umbral calculus as follows.

From (48) and (49), we have

$$
{ }_{B e l} B_{n}(x ; y) \sim\left(\frac{e^{t}-1}{t} e^{-y\left(e^{t}-1\right)}, t\right)
$$

and

$$
t_{\text {Bel }} B_{n}(x ; y)=n_{B e l} B_{n-1}(x ; y) .
$$

It follows from (52) that ${ }_{B e l} B_{n}(x ; y)$ is Appell for $\frac{e^{t}-1}{t} e^{-y\left(e^{t}-1\right)}$.

By (40) and (50), we have

$$
\begin{aligned}
\operatorname{Bel} B_{n}(x ; y) & =\frac{t}{e^{t}-1} e^{y\left(e^{t}-1\right)} x^{n}=e^{y\left(e^{t}-1\right)} B_{n}(x) \\
& =\sum_{k=0}^{n}\left(\begin{array}{l}
n \\
k
\end{array}\right) B e l_{k}(y) B_{n-k}(x),
\end{aligned}
$$

which is the special case of the result in (21). By (45) and (50), we also see that

$$
\begin{aligned}
B e l B_{n}(x ; y) & =\frac{t}{e^{t}-1} e^{y\left(e^{t}-1\right)} x^{n}=\frac{t}{e^{t}-1} \operatorname{Bel}_{n}(x ; y) \\
& =\sum_{k=0}^{\infty} \frac{B_{k}}{k !} t^{k} \operatorname{Bel}_{n}(x ; y)=\sum_{k=0}^{n}\left(\begin{array}{l}
n \\
k
\end{array}\right) B_{k} \operatorname{Bel}_{n-k}(x ; y) .
\end{aligned}
$$

We give the following theorem.

Theorem 18. For all $p(x) \in \mathbb{P}$, there exist constants $c_{0}, c_{1}, \ldots, c_{n}$ such that $p(x)=\sum_{k=0}^{n} c_{k}$ ${ }_{B e l} B_{k}(x ; y)$, where

$$
c_{k}=\frac{1}{k !}\left\langle\frac{e^{t}-1}{t} e^{-y\left(e^{t}-1\right)} t^{k} \mid p(x)\right\rangle .
$$


Proof. By (46), (48) and (51), we observe that

$$
\left\langle\frac{e^{t}-1}{t} e^{-y\left(e^{t}-1\right) t^{k}} \mid{ }_{B e l} B_{n}(x ; y)\right\rangle=n ! \delta_{n, k} \quad(n, k \geq 0),
$$

which yield the following relation

$$
\begin{aligned}
\left\langle\frac{e^{t}-1}{t} e^{-y\left(e^{t}-1\right)} t^{k} \mid p(x)\right\rangle & =\sum_{l=0}^{n} c_{l}\left\langle\frac{e^{t}-1}{t} e^{-y\left(e^{t}-1\right)} t^{k} \mid{ }_{B e l} B_{l}(x ; y)\right\rangle \\
& =\sum_{l=0}^{n} c_{l} l ! \delta_{l, k}=k ! c_{k},
\end{aligned}
$$

which gives the result in (53).

We give the following theorem.

Theorem 19. For $n>0$, we have

$$
{ }_{B e l} B_{n}(x ; y)=n \sum_{k=1}^{\infty} \sum_{l=0}^{k-1} \frac{y^{k}}{k !}\left(\begin{array}{c}
k-1 \\
l
\end{array}\right)(-1)^{k-1-l}(x+l)^{n-1} .
$$

Proof. From (40) and (50), we get

$$
\begin{aligned}
\mathrm{Bel}_{\mathrm{B}} B_{n}(x ; y) & =\frac{t}{e^{t}-1} e^{y\left(e^{t}-1\right)} x^{n}=\frac{t}{e^{t}-1} \sum_{k=0}^{\infty} y^{k} \frac{\left(e^{t}-1\right)^{k}}{k !} x^{n} \\
& =t \sum_{k=1}^{\infty} \sum_{l=0}^{k-1} \frac{y^{k}}{k !}\left(\begin{array}{c}
k-1 \\
l
\end{array}\right)(-1)^{k-1-l} e^{l t} x^{n} \\
& =n \sum_{k=1}^{\infty} \sum_{l=0}^{k-1} \frac{y^{k}}{k !}\left(\begin{array}{c}
k-1 \\
l
\end{array}\right)(-1)^{k-1-l}(x+l)^{n-1},
\end{aligned}
$$

which is the claimed result (54).

Here are some integral formulas by the following theorems.

Theorem 20. Let $p(x) \in \mathbb{P}$. We have

$$
\left\langle\frac{e^{t}-1}{t} e^{-y\left(e^{t}-1\right)} \mid p(x)\right\rangle=\sum_{k=0}^{\infty} \sum_{l=0}^{k+1}(-y)^{k}\left(\begin{array}{c}
k+1 \\
l
\end{array}\right)(-1)^{k-l+1} \int_{0}^{l} p(u) d u .
$$

Proof. By (41) and (42), we obtain the following calculations

$$
\begin{aligned}
\left\langle\frac{e^{t}-1}{t} e^{-y\left(e^{t}-1\right)} \mid x^{n}\right\rangle & =\frac{1}{n+1}\left\langle\frac{e^{t}-1}{t} e^{-y\left(e^{t}-1\right)} \mid t x^{n+1}\right\rangle \\
& =\frac{1}{n+1}\left\langle\sum_{k=0}^{\infty}(-y)^{k}\left(e^{t}-1\right)^{k+1} \mid x^{n+1}\right\rangle \\
& =\frac{1}{n+1} \sum_{k=0}^{\infty} \sum_{l=0}^{k+1}(-y)^{k}\left(\begin{array}{c}
k+1 \\
l
\end{array}\right)(-1)^{k-l+1}\left\langle e^{l t} \mid x^{n+1}\right\rangle \\
& =\frac{1}{n+1} \sum_{k=0}^{\infty} \sum_{l=0}^{k+1}(-y)^{k}\left(\begin{array}{c}
k+1 \\
l
\end{array}\right)(-1)^{k-l+1} l^{n+1} \\
& =\sum_{k=0}^{\infty} \sum_{l=0}^{k+1}(-y)^{k}\left(\begin{array}{c}
k+1 \\
l
\end{array}\right)(-1)^{k-l+1} \int_{0}^{l} x^{n} d x .
\end{aligned}
$$


Thus, from (55), we arrive at

$$
\left\langle\frac{e^{t}-1}{t} e^{-y\left(e^{t}-1\right)} t^{k} \mid p(x)\right\rangle=\sum_{k=0}^{\infty} \sum_{l=0}^{k+1}(-y)^{k}\left(\begin{array}{c}
k+1 \\
l
\end{array}\right)(-1)^{k-l+1} \int_{0}^{l} p(u) d u \quad(p(x) \in \mathbb{P}) .
$$

So, the proof is completed.

Example 1. If we take $p(x)={ }_{B e l} B_{n}(x ; y)$ in Theorem 20, on the one hand, we derive

$$
\begin{aligned}
\sum_{k=0}^{\infty} \sum_{l=0}^{k+1}(-y)^{k}\left(\begin{array}{c}
k+1 \\
l
\end{array}\right)(-1)^{k-l+1} \int_{0}^{l} B_{B e l} B_{n}(x ; y) d x & =\left\langle\frac{e^{t}-1}{t} e^{-y\left(e^{t}-1\right)} \mid B_{B e l} B_{n}(x ; y)\right\rangle \\
& =\left\langle 1 \mid \frac{e^{t}-1}{t} e^{-y\left(e^{t}-1\right)} \frac{t_{B e l} B_{n+1}(x ; y)}{n+1}\right\rangle \\
& =\frac{1}{n+1}\left\langle t^{0} \mid x^{n+1}\right\rangle=n ! \delta_{n+1,0} .
\end{aligned}
$$

On the other hand,

$$
\begin{aligned}
& (n+1) \sum_{k=0}^{\infty} \sum_{l=0}^{k+1}(-y)^{k}\left(\begin{array}{c}
k+1 \\
l
\end{array}\right)(-1)^{k-l+1} \int_{0}^{l} B e l B_{n}(x ; y) d x \\
= & (n+1) \sum_{k=0}^{\infty} \sum_{l=0}^{k+1}(-y)^{k}\left(\begin{array}{c}
k+1 \\
l
\end{array}\right)(-1)^{k-l+1} \sum_{u=0}^{n}\left(\begin{array}{l}
n \\
u
\end{array}\right) \text { Bel } B_{n-u}(y) \int_{0}^{l} x^{u} d x \\
= & \sum_{k=0}^{\infty} \sum_{l=0}^{k+1} \sum_{u=0}^{n} B e l B_{n-u}(y)\left(\begin{array}{c}
n+1 \\
u+1
\end{array}\right)\left(\begin{array}{c}
k+1 \\
l
\end{array}\right)(-y)^{k}(-1)^{k-l+1} l^{u+1},
\end{aligned}
$$

which yields the following interesting property for $n \geq 0$ :

$$
\sum_{k=0}^{\infty} \sum_{l=0}^{k+1} \sum_{u=0}^{n} B e l B_{n-u}(y)\left(\begin{array}{c}
n+1 \\
u+1
\end{array}\right)\left(\begin{array}{c}
k+1 \\
l
\end{array}\right)(-y)^{k}(-1)^{k-l+1} l^{u+1}=0 .
$$

Theorem 21. We have

$$
\left\langle\frac{e^{t}-1}{t} e^{-y\left(e^{t}-1\right)} \mid x^{n}\right\rangle=\int_{0}^{1} \operatorname{Bel}_{n}(u ;-y) d u .
$$

Proof. From (51) and (52), we write

$$
{ }_{B e l} B_{n}(x ; y)=\frac{t}{e^{t}-1} e^{y\left(e^{t}-1\right)} x^{n} \quad(n \geq 0) .
$$


By (41) and (42), we obtain the following calculations

$$
\begin{aligned}
\left\langle\frac{e^{t}-1}{t} e^{-y\left(e^{t}-1\right)\left|x^{n}\right\rangle}\right. & =\frac{1}{n+1}\left\langle\frac{e^{t}-1}{t} e^{-y\left(e^{t}-1\right)} \mid t x^{n+1}\right\rangle \\
& =\frac{1}{n+1}\left\langle\left(e^{t}-1\right) e^{-y\left(e^{t}-1\right)} \mid x^{n+1}\right\rangle \\
& =\frac{1}{n+1}\left\langle\sum_{k=0}^{\infty} \frac{\left(B e l_{k}(1 ;-y)-B e l_{k}(-y)\right)}{k !} t^{k} \mid x^{n+1}\right\rangle \\
& =\frac{1}{n+1} \sum_{k=0}^{\infty} \frac{\left(B e l_{k}(1 ;-y)-B e l_{k}(-y)\right)}{k !}(n+1) ! \delta_{n+1, k} \\
& =\frac{B e l_{n+1}(1 ;-y)-B e l_{n+1}(-y)}{n+1} \\
& =\int_{0}^{1} \operatorname{Bel}_{n}(u ;-y) d u .
\end{aligned}
$$

So, the proof is completed.

Theorem 22. Let $n$ be non-negative integer. Then, we have

$$
\left\langle\frac{e^{t}-1}{t} e^{-y\left(e^{t}-1\right)} \mid{ }_{B e l} B_{n}(x ; y)\right\rangle=\sum_{k=0}^{\infty} \sum_{l=0}^{k+1}(-y)^{k}\left(\begin{array}{c}
k+1 \\
l
\end{array}\right)(-1)^{k-l+1} \int_{l}^{l+1} B e l B_{n}(u+1 ; y) d u .
$$

Proof. By using (22) and (25) for $\alpha=1$, we obtain

$$
\int_{x}^{x+z}{ }_{B e l} B_{n}(u ; y) d u=\frac{1}{n+1}\left({ }_{B e l} B_{n+1}(x+z ; y)-{ }_{B e l} B_{n+1}(x ; y)\right) .
$$

Hence, by utilizing (52), we obtain

$$
\begin{gathered}
\left\langle\left.\frac{e^{t}-1}{t} e^{-y\left(e^{t}-1\right)}\right|_{B e l} B_{n}(x ; y)\right\rangle=\frac{1}{n+1}\left\langle\frac{e^{t}-1}{t} e^{-y\left(e^{t}-1\right)} \mid t_{B e l} B_{n+1}(x ; y)\right\rangle \\
=\frac{1}{n+1} \sum_{k=0}^{\infty} \sum_{l=0}^{k}(-y)^{k}\left(\begin{array}{c}
k \\
l
\end{array}\right)(-1)^{k-l}\left\langle\left.\left(e^{(l+1) t}-e^{l t}\right)\right|_{B e l} B_{n+1}(x ; y)\right\rangle \\
=\frac{1}{n+1} \sum_{k=0}^{\infty} \sum_{l=0}^{k+1}(-y)^{k}\left(\begin{array}{c}
k+1 \\
l
\end{array}\right)(-1)^{k-l+1}\left(B_{B e l} B_{n+1}(l+1 ; y)-{ }_{B e l} B_{n+1}(l ; y)\right) \\
=\sum_{k=0}^{\infty} \sum_{l=0}^{k+1}(-y)^{k}\left(\begin{array}{c}
k+1 \\
l
\end{array}\right)(-1)^{k-l+1} \int_{l}^{l+1} B e l B_{n}(u+1 ; y) d u .
\end{gathered}
$$

Comparing (56) with (57), we complete the proof.

The following theorem is useful for deriving any polynomial as a linear combination of the Bell-based Bernoulli polynomials.

Theorem 23. For $q(x) \in P_{n}$, let

$$
q(x)=\sum_{k=0}^{n} b_{k B e l} B_{k}(x ; y)
$$

where

$$
b_{k}=\sum_{m=0}^{\infty} \sum_{l=0}^{m}(-y)^{m}\left(\begin{array}{c}
m \\
l
\end{array}\right)(-1)^{m-l} \frac{\left(q^{(k-1)}(l+1)-q^{(k-1)}(l)\right)}{k !} .
$$


Proof. It follows from Theorem 18 that for $q(x) \in \mathbb{P}_{n}$, we have

$$
q(x)=\sum_{k=0}^{n} b_{k B e l} B_{k}(x ; y),
$$

with

$$
\left\langle\frac{e^{t}-1}{t} e^{-y\left(e^{t}-1\right)} t^{k} \mid q(x)\right\rangle=k ! b_{k} .
$$

Thus, from (58), we have

$$
\begin{aligned}
& b_{k}=\frac{1}{k !}\left\langle\left(e^{t}-1\right) e^{-y\left(e^{t}-1\right)} t^{k-1} \mid q(x)\right\rangle \\
& =\frac{1}{k !} \sum_{m=0}^{\infty} \sum_{l=0}^{m}(-y)^{m}\left(\begin{array}{c}
m \\
l
\end{array}\right)(-1)^{m-l}\left\langle\left(e^{(l+1) t}-e^{l t}\right) \mid t^{k-1} q(x)\right\rangle \\
& =\frac{1}{k !} \sum_{m=0}^{\infty} \sum_{l=0}^{m}(-y)^{m}\left(\begin{array}{c}
m \\
l
\end{array}\right)(-1)^{m-l}\left\langle\left(e^{(l+1) t}-e^{l t}\right) \mid q^{(k-1)}(x)\right\rangle \\
& =\frac{1}{k !} \sum_{m=0}^{\infty} \sum_{l=0}^{m}(-y)^{m}\left(\begin{array}{c}
m \\
l
\end{array}\right)(-1)^{m-l}\left(q^{(k-1)}(l+1)-q^{(k-1)}(l)\right) \text {. }
\end{aligned}
$$

Thus the proof is completed.

When we choose $q(x)=E_{n}(x)$, we have the following corollary, which is given by its proof.

Corollary 3. Let $n \geq 1$. We have

$$
\begin{gathered}
E_{n}(x)=\sum_{m=0}^{\infty} \sum_{l=0}^{m}(-y)^{m}\left(\begin{array}{c}
m \\
l
\end{array}\right)(-1)^{m-l}{ }_{B e l} B_{n}(x ; y) \\
+2 \sum_{k=0}^{n-1} \frac{(n)_{k-1}}{k !} \sum_{m=0}^{\infty} \sum_{l=0}^{m}(-y)^{m}(-1)^{m-l}\left(\begin{array}{c}
m \\
l
\end{array}\right)\left(l^{n-k+1}-E_{n-k+1}(l)\right){ }_{B e l} B_{k}(x ; y) .
\end{gathered}
$$

Proof. Recall that the Euler polynomials $E_{n}(x)$ are defined by $(c f .[12,23])$

$$
\sum_{n=0}^{\infty} E_{n}(x) \frac{t^{n}}{n !}=\frac{2}{e^{t}+1} e^{x t}
$$

which yields

$$
E_{n}(x) \sim\left(\frac{e^{t}+1}{2}, t\right) \quad(n \geq 0)
$$

and

$$
t E_{n}(x)=n E_{n-1}(x) .
$$

Set

$$
q(x)=E_{n}(x) \in \mathbb{P}_{n} .
$$

Then it becomes

$$
E_{n}(x)=\sum_{k=0}^{n} b_{k B e l} B_{k}(x ; y) .
$$


Let us now compute the coefficients $b_{k}$ as follows

$$
\begin{aligned}
& b_{k}=\frac{1}{k !}\left\langle\left(e^{t}-1\right) e^{-y\left(e^{t}-1\right) t^{k-1}} \mid E_{n}(x)\right\rangle \\
& =\frac{(n)_{k-1}}{k !}\left\langle\left(e^{t}-1\right) e^{-y\left(e^{t}-1\right)} \mid E_{n-k+1}(x)\right\rangle \\
& =\frac{(n)_{k-1}}{k !} \sum_{m=0}^{\infty} \sum_{l=0}^{m}(-y)^{m}\left(\begin{array}{c}
m \\
l
\end{array}\right)(-1)^{m-l}\left\langle\left(e^{(l+1) t}-e^{l t}\right) \mid E_{n-k+1}(x)\right\rangle \\
& =\frac{(n)_{k-1}}{k !} \sum_{m=0}^{\infty} \sum_{l=0}^{m}(-y)^{m}\left(\begin{array}{c}
m \\
l
\end{array}\right)(-1)^{m-l}\left(E_{n-k+1}(l+1)-E_{n-k+1}(l)\right) \text {. }
\end{aligned}
$$

Using $E_{1}(x)=x-\frac{1}{2}$ and

$$
E_{n}(x+1)-E_{n}(x)=2\left(x^{n}-E_{n}(x)\right),
$$

we have

$$
\begin{aligned}
E_{n}(x)= & b_{n B e l} B_{n}(x ; y)+\sum_{k=0}^{n-1} b_{k B e l} B_{k}(x ; y) \\
= & \sum_{m=0}^{\infty} \sum_{l=0}^{m}(-y)^{m}\left(\begin{array}{c}
m \\
l
\end{array}\right)(-1)^{m-l} B_{B e l} B_{n}(x ; y) \\
& +2 \sum_{k=0}^{n-1} \frac{(n)_{k-1}}{k !} \sum_{m=0}^{\infty} \sum_{l=0}^{m}(-y)^{m}(-1)^{m-l}\left(\begin{array}{c}
m \\
l
\end{array}\right)\left(l^{n-k+1}-E_{n-k+1}(l)\right){ }_{B e l} B_{k}(x ; y) .
\end{aligned}
$$

Recall from (18) that Bell-based Bernoulli polynomials of order $r \in \mathbb{N}_{0}$ are given by the following generating function:

$$
\sum_{n=0}^{\infty} B e l B_{n}^{(r)}(x ; y) \frac{t^{n}}{n !}=\frac{t^{r}}{\left(e^{t}-1\right)^{r}} e^{x t+y\left(e^{t}-1\right)} .
$$

If $t$ tends to 0 on the above, we have $B_{B e l} B_{0}^{(r)}(x ; y)=1$ that stands for $o\left(\frac{t^{r}}{\left(e^{t}-1\right)^{r}} e^{x t+y\left(e^{t}-1\right)}\right)=$ 0 . It means that the generating function of Bell-based Bernoulli polynomials of order $r$ is invertible and thus can be used as an application of Sheffer sequence.

Let

$$
g^{r}(t, x)=\frac{\left(e^{t}-1\right)^{r}}{t^{r}} e^{-y\left(e^{t}-1\right)} .
$$

Since $g^{r}(t, x)$ is an invertible series. It follows from (60) that ${ }_{B e l} B_{n}^{(r)}(x ; y)$ is Appell for $\left(\frac{e^{t}-1}{t}\right)^{r} e^{-y\left(e^{t}-1\right)}$. So, by (49), we have

$$
{ }_{B e l} B_{n}^{(r)}(x ; y)=\frac{1}{g^{r}(t, x)} x^{n}
$$

and

$$
t_{B e l} B_{n}^{(r)}(x ; y)=n_{B e l} B_{n}^{(r)}(x ; y) .
$$

Thus, we have

$$
\mathrm{Bel}_{n} B_{n}^{(r)}(x ; y) \sim\left(\frac{\left(e^{t}-1\right)^{r}}{t^{r}} e^{-y\left(e^{t}-1\right)}, t\right) .
$$

We give the following theorem.

Theorem 24. Let $n$ be non-negative integer. Then, we have 


$$
B_{B e l} B_{n}^{(r)}(x)=\sum_{i_{1}+\cdots+i_{r}=n}\left(\begin{array}{c}
n \\
i_{1}, \cdots, i_{r}
\end{array}\right) B_{B e l} B_{i_{r}}(x) \prod_{j=1}^{r-1} B_{i_{j}} .
$$

Proof. By (39) and (60), we get

$$
\left\langle\frac{t^{r}}{\left(e^{t}-1\right)^{r}} e^{z t+y\left(e^{t}-1\right)} \mid x^{n}\right\rangle={ }_{B e l} B_{n}^{(r)}(z ; y)=\sum_{l=0}^{n}\left(\begin{array}{c}
n \\
l
\end{array}\right){ }_{B e l} B_{n-l}^{(r)}(y) z^{l} .
$$

Here we find that

$$
\begin{aligned}
\left\langle\frac{t^{r}}{\left(e^{t}-1\right)^{r}} e^{y\left(e^{t}-1\right)} \mid x^{n}\right\rangle & =\left\langle\frac{t}{e^{t}-1} \times \cdots \times \frac{t e^{y\left(e^{t}-1\right)}}{e^{t}-1} \mid x^{n}\right\rangle \\
& =\sum_{i_{1}+\cdots+i_{r}=n}\left(\begin{array}{c}
n \\
i_{1}, \cdots, i_{r}
\end{array}\right){ }_{B e l} B_{i_{r}}(y) \times B_{i_{1}} \times \cdots \times B_{i_{r-1}} .
\end{aligned}
$$

By using (62), we have

$$
\left\langle\frac{t^{r}}{\left(e^{t}-1\right)^{r}} e^{y\left(e^{t}-1\right)} \mid x^{n}\right\rangle={ }_{B e l} B_{n}^{(r)}(y) .
$$

Therefore, by (63) and (64), we arrive at the desired result (61).

By setting $q(x)={ }_{B e l} B_{n}^{(r)}(x ; y) \in \mathbb{P}_{n}$ in Theorem 23, we provide the following Corollary.

Corollary 4. Let $n \in \mathbb{N}_{0}$ and $r \in \mathbb{N}_{0}$. Then

$$
{ }_{B e l} B_{n}^{(r)}(x ; y)=\sum_{k=0}^{n} \sum_{m=0}^{\infty} \sum_{l=0}^{m}\left(\begin{array}{c}
m \\
l
\end{array}\right)\left(\begin{array}{l}
n \\
k
\end{array}\right)(-y)^{m}(-1)^{m-l}{ }_{B e l} B_{n-k}^{(r-1)}(l ; y){ }_{B e l} B_{k}(x ; y) .
$$

Proof. By Theorem 23, we write

$$
{ }_{B e l} B_{n}^{(r)}(x ; y)=\sum_{k=0}^{n} b_{k B e l} B_{k}(x ; y),
$$

where the coefficient $b_{k}$ is given by

$$
\begin{aligned}
b_{k} & =\sum_{m=0}^{\infty} \sum_{l=0}^{m}(-y)^{m}\left(\begin{array}{c}
m \\
l
\end{array}\right)(-1)^{m-l} \frac{\left(q^{(k-1)}(l+1)-q^{(k-1)}(l)\right)}{k !} \\
& =\sum_{m=0}^{\infty} \sum_{l=0}^{m}(-y)^{m}\left(\begin{array}{c}
m \\
l
\end{array}\right)(-1)^{m-l} \frac{(n)_{k-1}}{k !}\left({ }_{B e l} B_{n-k+1}^{(r)}(l+1 ; y)-{ }_{B e l} B_{n-k+1}^{(r)}(l ; y)\right) .
\end{aligned}
$$

From (60), we have

$$
\begin{aligned}
\sum_{n=0}^{\infty}\left(B e l B_{n}^{(r)}(l+1 ; y)\right. & \left.-B_{e l} B_{n}^{(r)}(l ; y)\right) \frac{t^{n}}{n !}=\frac{t^{r}}{\left(e^{t}-1\right)^{r}} e^{l t+y\left(e^{t}-1\right)}\left(e^{t}-1\right) \\
& =t \frac{t^{r-1}}{\left(e^{t}-1\right)^{r-1}} e^{l t+y\left(e^{t}-1\right)} \\
& =\sum_{n=0}^{\infty} B e l B_{n}^{(r-1)}(l ; y) \frac{t^{n+1}}{n !} .
\end{aligned}
$$


By comparing the coefficients $t^{n}$ in the above equation, we get

$$
B_{B e l} B_{n}^{(r)}(l+1 ; y)-{ }_{B e l} B_{n}^{(r)}(l ; y)=n_{B e l} B_{n-1}^{(r-1)}(l ; y) .
$$

From (65), (66) and (67), the proof is completed.

The following theorem is useful for acquiring any polynomial as a linear combination of the Bell-based Bernoulli polynomials of order $r$.

Theorem 25. For $n \in \mathbb{N}_{0}$, we have

$$
q(x)=\sum_{k=0}^{n} b_{k B e l}^{r} B_{k}^{(r)}(x ; y) \in \mathbb{P}_{n}
$$

where

$$
b_{k}^{r}=\frac{1}{k !} \sum_{m=0}^{\infty} \sum_{l=0}^{m+r}(-y)^{m}\left(\begin{array}{c}
m+r \\
l
\end{array}\right)(-1)^{m+r-l} q^{(k-r)}(l) .
$$

Proof. Let us assume that

$$
q(x)=\sum_{k=0}^{n} b_{k B e l}^{r} B_{k}^{(r)}(x ; y) \in \mathbb{P}_{n} .
$$

We use a similar method in order to find the coefficient $b_{k}^{r}$ as same as Theorem 23. So we omit the details and give the following equality:

$$
\begin{aligned}
\left\langle\frac{\left(e^{t}-1\right)^{r}}{t^{r}} e^{-y\left(e^{t}-1\right)} t^{k} \mid q(x)\right\rangle & =\sum_{l=0}^{n} b_{l}^{r}\left\langle\frac{\left(e^{t}-1\right)^{r}}{t^{r}} e^{-y\left(e^{t}-1\right) t^{k}} \mid B_{B e l} B_{n}^{(r)}(x ; y)\right\rangle \\
& =\sum_{l=0}^{n} b_{l}^{r} l ! \delta_{l, k}=k ! b_{k}^{r}
\end{aligned}
$$

which gives for $k \geq r$,

$$
\begin{aligned}
b_{k}^{r} & =\frac{1}{k !}\left\langle\frac{\left(e^{t}-1\right)^{r}}{t^{r}} e^{-y\left(e^{t}-1\right) t^{k}|q(x)\rangle}\right. \\
& =\frac{1}{k !}\left\langle\sum_{m=0}^{\infty}(-y)^{m}\left(e^{t}-1\right)^{m+r} t^{k-r} \mid q(x)\right\rangle \\
& =\frac{1}{k !} \sum_{m=0}^{\infty} \sum_{l=0}^{m+r}(-y)^{m}\left(\begin{array}{c}
m+r \\
l
\end{array}\right)(-1)^{m+r-l}\left\langle e^{t l} \mid q^{(k-r)}(x)\right\rangle \\
& =\frac{1}{k !} \sum_{m=0}^{\infty} \sum_{l=0}^{m+r}(-y)^{m}\left(\begin{array}{c}
m+r \\
l
\end{array}\right)(-1)^{m+r-l} q^{(k-r)}(l) .
\end{aligned}
$$

Henceforth, by (68) and coefficient $b_{k}^{r}$, the proof is done.

Finally, we state the following Corollary:

Corollary 5. The following equality

$$
{ }_{B e l} B_{n}(x ; y)=\sum_{k=0}^{n} \sum_{m=0}^{\infty} \sum_{l=0}^{m+r}\left(\begin{array}{c}
m+r \\
l
\end{array}\right)(-y)^{m}(-1)^{m+r-l} \frac{(n)_{k-r}}{k !} B_{B e l} B_{n-k+r}(l ; y){ }_{B e l} B_{k}^{(r)}(x ; y)
$$

holds for $n, r \in \mathbb{N}_{0}$, 
Proof. Let us consider $q(x)={ }_{B e l} B_{n}(x ; y) \in \mathbb{P}_{n}$. Then, by Theorem 25, we have

$$
{ }_{B e l} B_{n}(x ; y)=\sum_{k=0}^{n} b_{k B e l}^{r} B_{k}^{(r)}(x ; y) .
$$

From Theorem 25 and (69), after some basic computations, we arrive at the claimed result.

\section{Conclusions}

In the present paper, we have considered Bell-based Stirling polynomials of the second kind and derived some useful relations and properties including some summation formulas related to the Bell polynomials and Stirling numbers of the second kind. Then, we have introduced Bell-based Bernoulli polynomials of order $\alpha$ and have investigated multifarious correlations and formulas including some summation formulas and derivative properties. Also, we have acquired diverse implicit summation formulas and symmetric identities for Bell-based Bernoulli polynomials of order $\alpha$. Moreover, we have analyzed some special cases of the results. Furthermore, we have attained several interesting formulas of Bellbased Bernoulli polynomials of order $\alpha$ arising from umbral calculus to have alternative ways of deriving our results.The results obtained in this paper are generalizations of the many earlier results, some of which are involved in the related references in [1-23]. For future directions, we will consider that the polynomials introduced in this paper can be examined within the context of the monomiality principle.

Author Contributions: All authors contributed equally to this work. All authors have read and agreed to the published version of the manuscript.

Funding: This research received no external funding.

Acknowledgments: We would like to thank the reviewers for their careful reading our manuscript, which have improved the paper substantially.

Conflicts of Interest: The authors declare no conflict of interest.

\section{References}

1. Acikgoz, M.; Ates, R.; Duran, U.; Araci, S. Applications of $q$-Umbral Calculus to Modified Apostol Type $q$-Bernoulli Polynomials. J. Math. Stat. 2018, 14, 7-15. [CrossRef]

2. Benbernou, S.; Gala, S.; Ragusa, M.A. On the regularity criteria for the 3D magnetohydrodynamic equations via two components in terms of BMO space. Math. Methods Appl. Sci. 2014, 37, 2320-2325. [CrossRef]

3. Boas, R.P.; Buck, R.C. Polynomial Expansions of Analytic Functions; Springer: Berlin/Gottingen/Heidelberg, Germany, 1958.

4. Carlitz, L. Some remarks on the Bell numbers. Fibonacci Quart. 1980, 18, 66-73.

5. Dere, R.; Simsek, Y.; Srivastava, H.M. A unified presentation of three families of generalized Apostol type polynomials based upon the theory of the umbral calculus and the umbral algebra. J. Number Theory 2013, 133, 3245-3263. [CrossRef]

6. Dere, R.; Simsek, Y. Applications of umbral algebra to some special polynomials. Adv. Stud. Contemp. Math. 2012, $22,433-438$.

7. Dere, R.; Simsek, Y. Hermite base Bernoulli type polynomials on the umbral algebra. Russ. J. Math. Phys. 2015, 22, 1-5. [CrossRef]

8. Khan, S.; Pathan, M.A.; Hassan, N.A.M.; Yasmin, G. Implicit summation formulae for Hermite and related polynomials. J. Math. Anal. Appl. 2008, 344, 408-416. [CrossRef]

9. Kim, D.S.; Kim, T. Some identities of Bell polynomials. Sci. China Math. 2015, 58, 2095-2104. [CrossRef]

10. Kim, T.; Kim, D.S.; Jang, G.-W.; Jang, L.C. A generalizations of some results for Appell polynomials to Sheffer polynomials. J. Comput. Anal. Appl. 2019, 26, 889-898.

11. Kim, D.S.; Kim, T. Umbral calculus associated with Bernoulli polynomials. J. Number Theory 2015, 147, 871-882. [CrossRef]

12. Kim, D.S.; Kim, T.; Lee, S.-H.; Rim, S.-H. Some identities of Bernoulli, Euler and Abel polynomials arising from umbral calculus. Adv. Differ. Equ. 2013, 2013, 15. [CrossRef]

13. Kim, T.; Kim, D.S.; Jang, L.-C.; Lee, H.; Kim, H.-Y. Complete and incomplete Bell polynomials associated with Lah-Bell numbers and polynomials. Adv. Differ. Equ. 2021, 2021, 101. [CrossRef]

14. Kim, T.; Kim, D.S.; Kwon, H.-I.; Rim, S.-H. Some identities for umbral calculus associated with partially degenerate Bell numbers and polynomials. J. Nonlinear Sci. Appl. 2017, 10, 2966-2975. [CrossRef]

15. Kim, T.; Kim, D.S.; Jang, G.-W.; Kwon, J. Fourier series of higher-order ordered Bell functions. J. Nonlinear Sci. And Applications 2017, 10, 3851-3855. [CrossRef] 
16. Kim, T.; Kim, D.S.; Jang, G.-W.; Jang, L.-C. Degenerate ordered Bell numbers and polynomials associated with umbral calculus. J. Nonlinear Sci. Appl. 2017, 10, 5142-5155. [CrossRef]

17. Kim, T.; Kim, D.S.; Dolgy, D.-V.; Kwon, J. Fourier series of finite product of Bernoulli and ordered Bell functions. J. Nonlinear Sciences Appl. 2018, 11, 500-515. [CrossRef]

18. Kim, T.; Kim, D.S.; Kim, H.Y.; Kwon, J. Some identities of degenerate Bell polynomials. Mathematics 2020, 8, 40. [CrossRef]

19. Kucukoglu, I.; Simsek, B.; Simsek, Y. Generating functions for new families of combinatorial numbers and polynomials: approach to Poisson-Charlier polynomials and probability distribution function. Axioms 2019, 8, 112. [CrossRef]

20. Pathan, M.A.; Khan, W.A. Some implicit summation formulas and symmetric identities for the generalized Hermite-Bernoulli polynomials. Mediterr. J. Math. 2015, 12, 679-695. [CrossRef]

21. Roman, S. The Umbral Calculus; Academic Press, Inc.: New York, NY, USA, 1984.

22. Srivastava, H.M.; Choi, J. Zeta and q-Zeta Functions and Associated Series and Integrals; Elsevier Science Publishers: Amsterdam, The Netherlands, 2012; 674 p.

23. Srivastava, H.M.; Pinter, A. Remarks on some relationships between the Bernoulli and Euler polynomials. Appl. Math. Lett. 2004, 17, 375-380. [CrossRef]

24. Srivastava, H.M.; Manocha, H.L. A Treatise on Generating Functions; Ellis Horwood Limited. Co.: New York, NY, USA, 1984.

25. Wang, W.; Wang, T. Identities on Bell polynomials and Sheffer sequences. Discret. Math. 2009, 309, 1637-1648. [CrossRef]

26. Bell, E.T. Exponential polynomials. Ann. Math. 1934, 35, 258-277. [CrossRef] 